\title{
Viscous Populations and Their Support for Reciprocal
}

\section{Cooperation}

\author{
James A. R. Marshall*
}

Complex Systems Modelling Group, Department of Earth Science and Engineering, Imperial College London, SW7 2AZ, UK

James.A.Marshall@imperial.ac.uk

+44(0)2075947493

Jonathan E. Rowe

School of Computer Science, University of Birmingham, B15 2TT, UK

J.E.Rowe@cs.bham.ac.uk

+44(0)1214142985

* Corresponding author

\begin{abstract}
Viscous populations, those whose members are spatially distributed and have limited mobility and locality of interaction and mating, have been proposed to support the evolution of reciprocal cooperation among self-interested individuals. Here we present a model of such a population and describe how its examination yielded the realisation that different classes of viscous populations exist with differing levels of support for reciprocal cooperation. Specifically we find from our model that in a spatially distributed population with increased viscosity, the reciprocally cooperative Tit-for-Tat strategy may not be globally stable, due to a corresponding increase in local population density.
\end{abstract}




\section{Introduction: Reciprocal Cooperation in Viscous Populations}

The search for explanations of cooperative behaviour between self-interested individuals has a long and fruitful history. One of the prime explanations proposed for the occurrence of cooperation in diverse populations throughout the natural world is reciprocal cooperation $[2,16]$. Reciprocal cooperation suggests that cooperation can become prevalent in populations; if individuals are able to remember others' behaviour, and if interactions between the same individuals are sufficiently frequent to make it more profitable, in the long-term, to establish a mutually cooperative relationship than to attempt to establish an exploitative one. Axelrod [2] identified Rapoport's reciprocally cooperative strategy Tit-for-Tat, which reciprocates its opponents' cooperation or defection actions one-for-one, as being highly successful in such situations. Axelrod also used the concept of an Evolutionary Stable Strategy from evolutionary game theory $[12,13]$ to establish a collective stability condition for the strategy Tit-for-Tat, showing when Tit-for-Tat will be superior in a population according to its performance against two strategies; the strategy of unconditional defection and the strategy of alternating cooperation with defection.

Suitable conditions for reciprocal cooperation to occur under have previously been proposed by Trivers [16] as being found in spatially distributed populations with limited mobility and locality of interaction between individuals. Such populations can be given the name "viscous populations" [6]. Whereas other models of spatial populations have focused on persistent cooperation or altruism due to, for example, dynamic coexistence [14], ecological symmetry breaking [4], or hypercycles [3], here we present a model expected to support cooperation through increased interaction length due to population viscosity. 
In this paper we will briefly present the design of the model in section 2 , then in sections 3 and 4 we will describe how to assess the degree of support the model provides for reciprocal cooperation, using the criterion of Tit-for-Tat's collective stability. In section 5 we will generalise these results and in section 6 make some observations about the usefulness of the Artificial Life methodology.

\section{A Viscous Population Cooperative Model}

To study, among other things, reciprocal cooperation in viscous populations, we developed an agent-based simulation model of an abstract population ${ }^{1}$. This model has already been described in detail elsewhere $[10,11]$ and is only briefly described here.

The model comprises a population of individuals and an environment in which they are situated. The environment is simply a grid of cells, with each edge of the grid wrapped around to meet its opposite edge to form a torus, in order to eliminate edge effects. Each cell is capable of housing any number of individuals from zero upwards. At each time step, individuals are able to move to any of the eight adjacent cells with a certain probability $m$. This value $m$ is the main parameter for variation in the model and inversely corresponds with "viscosity". Cells are used as the local area of interaction, that is individuals can only interact with other individuals in the cell they currently inhabit. Interaction between individuals occurs with the random formation of pairs within cells at each time step and takes the form of a single round of the Iterated Prisoner's Dilemma (IPD). Individuals have both a memory of their interactions with other individuals and a strategy that specifies the current action choice in the IPD, cooperate or defect, based on the action choices of both participants

\footnotetext{
${ }^{1}$ The source code for the model is downloadable from ftp://ftp.swarm.org/pub/swarm/apps/objc/contrib/EPD-2.1.1-2.1.1.tar.gz
} 
on their last interaction together. The model is an evolutionary one, with selection pressure applied on performance in IPD interactions through the imposition of a per time step living cost, so individuals die and are replaced by the offspring of the individuals in the population that score higher in IPD interactions. Individuals are also terminated with a random death probability $d$ at each time step, to prevent successful individuals becoming "immortal". Reproduction is sexual and uses parameterised uniform crossover and point mutation operators to combine two parents' chromosomes into an offspring chromosome. The chromosome encoding follows Mar \& St Denis [9], but with a binary alphabet, to specify a two-dimensional strategy. Thus five loci are used (one for each potential interaction history, one for the first interaction), each with two alleles (cooperate or defect). Offspring are placed in the same cell as their parents.

In any computer simulation model the update mechanism requires careful consideration. Huberman \& Glance [7] examined the effects of synchronous and asynchronous update schemes on the behaviour of cellular automata, concluding that the behaviour could in fact be highly contingent on the update scheme used. Although asynchronous update is unlikely to be so significant for a model such as that described above, care does need to be taken in avoiding bias through arbitrary ordering of updates. In particular, the order of updates during reproduction is significant in our model: those individuals receiving their reproduction opportunity later in a simulation time step will have a lower probability of actually reproducing, as other individuals reproducing earlier in the time step consume the environment's remaining carrying capacity. To avoid such problems, our model divides each simulation time step into several phases, such as movement, interaction and reproduction. During each phase 
individuals are updated in a series of "microsteps" [7], with the update order randomised each time.

\section{A Simple Analysis of Interaction Probability in the Model}

Marshall \& Rowe [10] presented a simple analysis of the probability of two consecutive interactions between the same agents in the model described above, the details of which are presented here. In the following equations, $d$ is the environmental death probability, $m$ is the agent movement probability, and $p$ is the average cell population size for populated cells.

Cells are used as the local area for interaction, therefore in order for two agents that interact in one time step to interact in the following time step, both must end up occupying the same cell in the environment. As the agents interacted in the first place they are necessarily in the same cell to begin with. Then there are two possible ways in which they can end up occupying the same cell in the following time step: both agents can remain in the same cell, or both agents can move to the same neighbouring cell. Therefore the probability of both agents ending up in the same cell on the next time step is

$$
(1-m)^{2}+\frac{m^{2}}{8}
$$

where the first term corresponds to the probability that both agents remain in the same cell, and the second term corresponds to the probability that both agents move to the same cell in their Moore neighbourhood of eight cells.

Assuming that both agents end up in the same cell, in order to interact both agents must survive the random death in the environment, and must also be formed into an interaction pair during the random process of pairing agents in a cell's population. Thus the probability of both agents interacting together once they are in the same cell is 


$$
\frac{(1-d)^{2}}{p-1}
$$

Then given one interaction between a pair of agents, the probability that the same pair of agents will interact on the following time step is given by

$$
u=\frac{(1-d)^{2}\left((1-m)^{2}+\frac{m^{2}}{8}\right)}{p-1}
$$

The probability of repeated interaction given by $u$ is a simplification of that actual probability in two respects. Firstly, it ignores the possibility of agent death through lack of energy, which occurs when an agent is unable to generate enough energy from its IPD interactions with other agents to cover the environmental cost of living. Taking account of this possibility is very difficult and for simplicity it is ignored here. Secondly, the calculation of $u$ does not account for the possibility of agents interacting again at some time step in the future other than the next time step. Taking account of this possibility becomes more complicated the more time steps in the future are considered, and again for simplicity it is ignored here. As will be shown later, such simplifications are not significant for the argument we wish to make in this paper. For now it is enough to note from $p$ 's position in the denominator of (3) that as the local population size $p$ in a cell increases, so the probability $u$ of continued interaction between any two given individuals decreases.

\section{Evaluating Support for Reciprocal Cooperation in the Model}

Axelrod's [2] collective stability condition for Tit-for-Tat is based on the probability $w$ of another interaction between two individuals occurring after the current interaction, also known as the "shadow of the future". Given the Prisoner's Dilemma payoffs: $T$ (the temptation to defect when an opponent cooperates), $R$ (the reward for mutual cooperation), $P$ (the punishment for mutual defection), and $S$ (the sucker's 
payoff from cooperating when an opponent defects), then Tit-for-Tat is collectively stable when

$$
w>\max \left[\frac{(T-R)}{(T-P)}, \frac{(T-R)}{(R-S)}\right]
$$

This can be broken down into two separate requirements. The first requirement $w>(T$ $-R) /(T-P)$ is the requirement that Tit-for-Tat cannot be invaded by the strategy of unconditional defection, All-D. The second requirement $w>(T-R) /(R-S)$ is the requirement that Tit-for-Tat cannot be invaded by the strategy that alternates defection with cooperation, which shall be called Alternating-D-C for the purposes of this discussion. These two strategies were determined by Axelrod to be the two representative strategies against which Tit-for-Tat should be tested for collective stability.

The reciprocal cooperative strategy Tit-for-Tat is one of the most robust and successful strategies in the IPD without noise [2], which is the interaction model used in this work. Therefore Axelrod's condition for the collective stability of Tit-for-Tat is used here as a guide to assessing the degree to which reciprocal cooperation is supported in the model under the parameters studied. Evaluating the collective stability of Tit-for-Tat, using the Prisoner's Dilemma payoffs $T=5, R=3, P=1$ and $S=0$ from the model, gives the following conditions; $w>0.5$ for the collective stability of Tit-for-Tat against All-D and $w>0.67$ for the collective stability of Titfor-Tat against Alternating-D-C. Hence the requirement for the collective stability of Tit-for-Tat is $w>0.67$.

As $w$ is the probability of repeated interaction, the expected number of interactions is given by 


$$
v=\frac{1}{(1-w)}
$$

This means the minimum number of interactions required for the collective stability of Tit-for-Tat in the model is 3.

\section{Clustering Viscous Populations}

By taking (3) from the preceding section and recognising that $u$ is an estimate of $w$, we can evaluate the maximum required local cell population size $p^{\prime}$ for an expected shadow of the future $w$, given any $m$ and $d$, as follows

$$
p^{\prime}=\frac{(1-d)^{2}\left((1-m)^{2}+\frac{m^{2}}{8}\right)}{w}+1
$$

Taking the minimum requirement for the collective stability of Tit-for-Tat as the expected shadow of the future gives $w=0.67$. Then, with death probability $d=0.05$ from the model studied, equation (6) can be used to calculate the maximum average local population size required for the collective stability of Tit-for-Tat under varying movement probability $m$, as shown below in figure 1 .

<figure 1 about here>

Note that as population viscosity increases ( $m$ decreases) in the model the maximum local population size for the stability of Tit-for-Tat also increases. However, at the same time as population viscosity increases, average local population size per cell for 
inhabited cells increases, as shown below in figure $2^{2}$. This is because in our model low viscosity populations tend to spread over a large area, whereas high viscosity causes local clustering, due to the per time-step living cost which requires that agents engage in continued social interaction with each other in order to survive. Note that the population is approximately uniformly distributed over the inhabited cells of the environment, as indicated by the low standard deviation of population size for such cells shown in figure 2 . At low population viscosity $(m=1)$ the population covers almost the entire grid, whereas at high population viscosity $(m=0.1)$ inhabited cells only cover approximately $50 \%$ of the grid.

\section{<figure 2 about here>}

From figure 2 we expect that, under these parameter settings, reciprocal cooperation is not well supported. The actual local population size already exceeds the maximum required for the collective stability of Tit-for-Tat at low population viscosity $(m=1)$ and diverges from the maximum requirement as population viscosity increases. This prediction is supported by the low average number of interactions observed in the model, which only approaches 1.5 at $m=0.1$ [10], far below the minimum required for Tit-for-Tat to be collectively stable. This extends our previous consideration of the model [10] by showing that in fact reciprocal cooperation may not be, by itself, a

\footnotetext{
${ }^{2}$ Results averaged over 20 runs with the following parameters: horizontal environment size in cells $=$ 10 , vertical environment size in cells $=10$, initial agent energy $=15$, energy living cost $=3$, crossover rate $=0.1$, mutation rate $=0.01$, maximum population size $=200$, initial population size $=75$, death probability $d=0.05$. Prisoner's Dilemma payoffs used were as described in the text $(T=5, R=3, S=$ $0, P=1)$. Simulation runs were terminated after 1000 time-steps; local population size for inhabited cells, and number of interactions, were averaged over the duration of each run.
} 
good explanation for observed increases in cooperation as the model population becomes more viscous.

It is possible to generalise this result to a result for viscous populations in which increased viscosity also leads to an increase in the local population density experienced by individuals in that population, of which the model presented here is an example. To do this it is necessary to treat the estimated repeat interaction probability (3), presented above, as a guide to the actual average number of interactions in the population. Equation (3) gives an indication of the probability of another interaction between two individuals in the next time step, given they interact in the current time step. There are two sources of error in the estimate given by (3), the first leading to an underestimate by discounting interactions at more than one time step in the future, the second leading to an overestimate by discounting the possibility of one or both of the interacting individuals running out of energy and dying. For the purposes of our argument we can ignore the first source of error, the consistent underestimation of $u$ through not considering repeat interactions more than one time step in the future, as intuitively if the probability of repeat interaction on the next time step is increased, then the probability of another interaction occurring between the same two individuals at any time step in the future is also increased. Similarly we can ignore the second source of error, the variable overestimation of $u$ due to not taking death through lack of energy into account, because, as will become clear, the focus of our argument is on the change in $u$ under varying $m$, due to change in local population size $p$. Although death through lack of energy may become more or less significant at different $m$ this does not affect the main argument of this paper.

We now conclude our argument as follows. The repeat interaction probability (3) has been used to estimate the maximum average local population size required for the 
collective stability of Tit-for-Tat, presented in figure 1 above. As can be seen from figure 1, the maximum average local population size required for the collectivestability of Tit-for-Tat increases as population viscosity increases. At the same time, viscous populations such as that implemented in the model exhibit an increase in average local population size as population viscosity increases, as shown in figure 2 . The question of how much support is provided for reciprocal cooperation in such a viscous population therefore rests on the initial levels and the relative rates of increase of the average local population size, and of the maximum local population size required for the collective stability of Tit-for-Tat, as population viscosity increases.

We label populations where local population density increases with reduced mobility "clustering" viscous populations. Such populations exhibit an inhibiting force on support for reciprocal cooperation, in contrast with "non-clustering" viscous populations where a fixed or bounded local population density will not give rise to such a force. This clustering effect is particularly likely to be observed in populations that are subject to the Allee effect [1], in which individual members of the population are dependent on the presence in their vicinity of other individuals from the same population in order to survive.

Fundamentally the increase in local population size experienced by individuals in the model is due to its spatial granularity. The smallest spatial unit in the model is a single cell, within which spatiality is lost and a local panmictic population exists. The size of this spatial unit in the model corresponds, in the real world, to distances over which interactions can take place, and physical constraints on individuals inhabiting the same region. If these factors allow local population size to increase significantly then some form of social mechanism for limiting the number of interactants an individual 
experiences may be required for reciprocal cooperation to become established. Such a mechanism goes beyond the scope of the model presented here.

Perhaps most natural populations are of the "clustering" type, although examples of "non-clustering" natural populations doubtless exist with behaviours that resist overcrowding, such as territoriality or living in groups of limited size. Sessile organisms such as plants would seem to fall into the latter category while, for example species of ungulates can exhibit a range of group sizes, from solitary individuals to group living in herds of up to 1000 . However, dominance hierarchies in such herds could provide a social mechanism, of the type described above, to limit number of interactants experienced by any individual in the population [8].

As a final aside, we feel that this inhibiting force on reciprocal cooperation in certain kinds of viscous population provides a pleasing companion to the proposed force inhibiting kin selection in viscous populations, namely increased competition between relatives [17].

\section{Discussion}

The argument put forward in this letter, that viscous populations that cluster and so have increased local population density may not provide complete support for reciprocal cooperation, is an intuitive one. After reading this letter we expect the reader will see why it is obvious that this should be so. However, we believe that obtaining this a posteriori knowledge required the formulation and observation of the model described. The original argument for viscous populations to support reciprocal cooperation [16] was in the form of a simple thought experiment. The approach here has been to take that thought experiment and examine the implications of its original formulation through computer simulation. This is a simple example of what Di Paolo et al [5] term the "opaque thought experiment", in which the implications of the 
assumptions underlying the model cannot necessarily be arrived at by the unaided human mind. Thus a simulation model of the subject of the thought experiment is constructed, and observation and explanation of the model precipitate modification of the theory being modelled. As identified by Taylor \& Jefferson [15], one of the key strengths of the individual-based computer simulation approach lies in the relaxation of assumptions that are inherent in top-down equation-based approaches to such modelling. In the example we have presented here we feel it is clear that an attempt to model a viscous clustering population in a descriptive manner would require an a priori insight on the part of the modeller that such populations could become denser under increased viscosity, whereas in the approach used here the insight arises through simulation and observation of the model. We agree with Di Paolo et al and Taylor \& Jefferson that the use of computer simulation modelling in this manner is a hallmark of Artificial Life research.

\section{Acknowledgements}

We thank S. Bullock and three anonymous reviewers for constructive comments on an earlier draft of this letter. We also thank A. Dornhaus and N. Franks for discussions on real examples of clustering and non-clustering populations.

\section{References}

1. Allee, W. C. (1931) Animal aggregations: a study in general sociology. Chicago: University of Chicago Press.

2. Axelrod, R. (1984) The evolution of cooperation. New York: Basic Books.

3. Boerlijst, M. \& Hogeweg, P. (1991) Self-structuring and selection: spiral waves as a substrate for prebiotic evolution. In C. G. Langton, C. Taylor, J. Doyne Farmer \& S. Rasmussen (Eds.), Artificial life II (pp. 255-276). Redwood City, CA: Addison-Wesley 
4. Di Paolo, E. A. (2000) Ecological symmetry breaking can favour the evolution of altruism in an action-response game. Journal of Theoretical Biology, 203, $135-152$.

5. Di Paolo, E. A., Noble, J. \& Bullock, S. (2000) Simulation models as opaque thought experiments. In M. Bedau, J. McCaskill \& S. Packard (Eds.), Proceedings of the seventh international conference on artificial life (pp. 497506). Cambridge MA: MIT Press.

6. Hamilton, W. D. (1964) The genetical evolution of social behaviour I. Journal of Theoretical Biology, 7, 1-16.

7. Huberman, B. \& Glance, N. (1993) Evolutionary games and computer simulations. Proceedings of the National Academy of Sciences of the United States of America, 90, 16, 7715-7718.

8. Jarman, P. J. (1974) The social organisation of antelope in relation to their ecology. Behaviour, 48, 215-267.

9. Mar, G. \& St Denis, P. (1994) Chaos in cooperation: continuous-valued prisoner's dilemmas in infinite-valued logic. International Journal of Bifurcation and Chaos, 4, 4, 943-958.

10. Marshall, J. A. R. \& Rowe, J. E. (2000) Investigating the mechanisms underlying cooperation in viscous population multi-agent systems. In M. Bedau, J. McCaskill \& S. Packard (Eds.), Proceedings of the seventh international conference on artificial life (pp. 348-352). Cambridge MA: MIT Press.

11. Marshall, J. A. R. \& Rowe, J. E. (2003) Kin selection may inhibit the evolution of reciprocation. To appear in Journal of Theoretical Biology. 
12. Maynard Smith, J. (1982) Evolution and the theory of games. Cambridge: Cambridge University Press.

13. Maynard Smith, J. \& Price, G. R. (1973) The logic of animal conflict. Nature, $246,15-18$.

14. Nowak, M. \& May, R. (1992) Evolutionary games and spatial chaos. Nature, $359,826-829$.

15. Taylor, C. \& Jefferson, D. R. (1995) Artificial life as a tool for biological inquiry. In C. Langton (Ed.), Artificial life: an overview (pp.1-14). Cambridge, MA: MIT Press.

16. Trivers, R. (1971) The evolution of reciprocal altruism. Quarterly Review of Biology, 46, 35-57.

17. Wilson, D. S., Pollock, G. B. \& Dugatkin, L. A. (1992) Can altruism evolve in purely viscous populations? Evolutionary Ecology, 6, 331-341. 


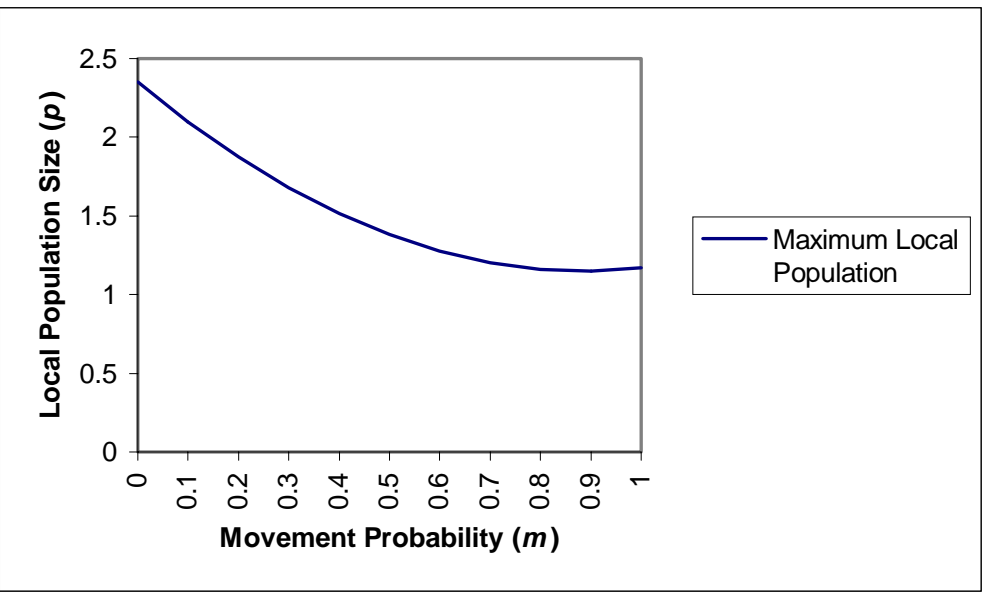

Figure 1. Maximum Local Population Size Required for Collective Stability of Tit-for-

Tat in the Model 


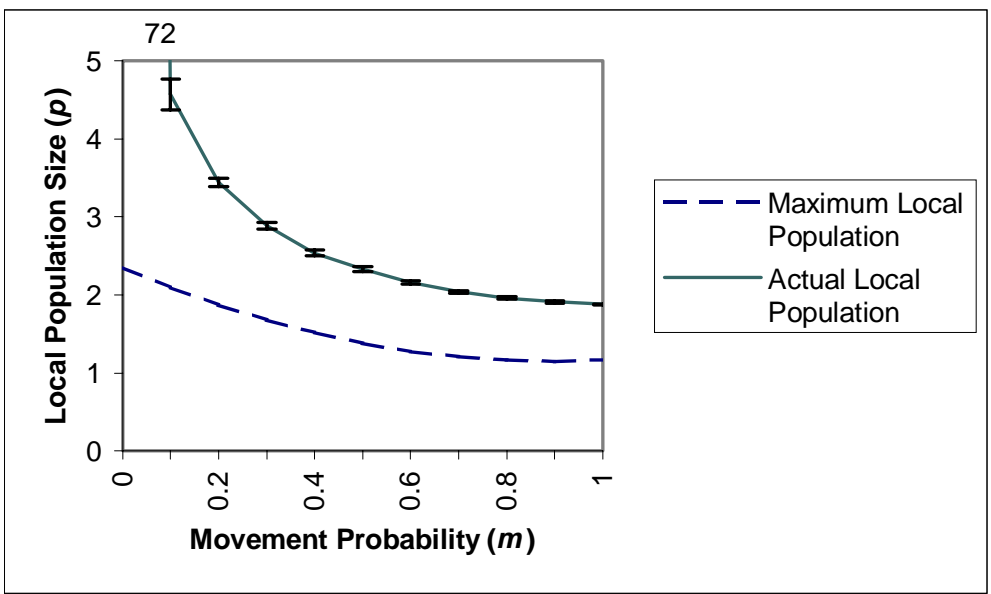

Figure 2. Experimental Results for Actual Local Population Size in the Model 